\title{
Piston Head Device
}

National Cancer Institute

\section{Source}

National Cancer Institute. Piston Head Device. NCI Thesaurus. Code C49981.

The portion of a piston above the topmost seal ring. 